\title{
Niveau de contamination des produits maraîchers par les substances toxiques sur le site de Houéyiho au Bénin
}

\author{
V. AGUEH ${ }^{1}$, C.C. DEGBEY ${ }^{1 *}$, C. SOSSA-JEROME ${ }^{1}$, D. ADOMAHOU ${ }^{1}$, \\ M.N. PARAISO ${ }^{1,}$ S. VISSOH ${ }^{2}$, M. MAKOUTODE ${ }^{1}$ et B. FAYOMI ${ }^{3}$ \\ ${ }^{1}$ Institut Régional de Santé Publique de Ouidah, Université d'Abomey Calavi, Bénin ; BP 384 Ouidah, Bénin. \\ ${ }^{2}$ Ministère de la Santé, Bénin. \\ ${ }^{3}$ UER de Santé au Travail et Environnement, Faculté des Sciences de la Santé de Cotonou, Bénin. \\ *Auteur correspondant; E-mail : comlancy@yahoo.fr
}

\section{RESUME}

Il est admis que l'utilisation inappropriée des intrants agricoles et des pesticides a des effets sur la qualité des produits maraîchers. C'est pour étudier le niveau de contamination des produits maraîchers sur le site de Houéyiho que la présente étude a été menée. Il s'agit d'une étude transversale descriptive et analytique qui a opté pour une approche éco systémique basée sur la transdisciplinarité, la participation des producteurs et le genre/éthique. Des prélèvements de produits maraîchers (Daucus carota, Vernonia amygdalina, Lactuca sativa), d'eau et de sol ont été effectués à différents niveaux du site. Les résultats de cette étude révèlent que le niveau de contamination des produits maraîchers est élevé en zinc $(198,84 \mathrm{mg} / \mathrm{kg})$, en plomb $(17,99 \mathrm{mg} / \mathrm{kg})$, en fer $(1042,33 \mathrm{mg} / \mathrm{kg})$ et en cuivre $(11,02 \mathrm{mg} / \mathrm{kg})$ par rapport aux normes exigées par CODEX STAN 1931995 révisé en 2007 et qui sont respectivement de $5 \mathrm{mg} / \mathrm{kg}, 0,3 \mathrm{mg} / \mathrm{kg}, 2 \mathrm{mg} / \mathrm{kg}, 3 \mathrm{mg} / \mathrm{kg}$. Des efforts restent à fournir pour réduire davantage ces concentrations à travers des sensibilisations, à l'utilisation effective du compost fabriqué sur le site pour minimiser les risques encourus par la population.

(C) 2015 International Formulae Group. All rights reserved.

Mots clés : Substances toxiques, cultures maraîchères, milieu urbain, Bénin.

\section{INTRODUCTION}

La qualité des denrées alimentaires présentes sur le marché tend ces dernières années à ne plus être prise en considération par les consommateurs dans les pays développés. Le maraîchage est une activité génératrice de revenus qui se développe de plus en plus dans les grandes villes africaines en raison de la forte demande pour la consommation dans les ménages et dans les hôtels (Gnandli et al., 2006 ; Sanny, 2002).

Le maraîchage urbain est considéré en Afrique comme une solution aux problèmes d'approvisionnement en légume des villes de plus en plus peuplées. Cette agriculture intensive est très dynamique, mais elle n'est pas sans danger. En effet, la main-d'œuvre, peu ou pas qualifiée, manipule des produits délicats (engrais et pesticides). Si la quantité est au rendez-vous, la qualité l'est moins, car les traitements sont souvent mal conduits (Madjouma et al., 2009). Les cultures maraîchères constituent donc la principale activité de l'agriculture urbaine répondant de façon efficace à la demande alimentaire urbaine. Le maraîchage contribue ainsi à la 
sécurité alimentaire et à la réduction de la pauvreté des ménages, en particulier celle des femmes (Madjouma et al., 2009 ).

$\mathrm{Au}$ Benin, le taux d'urbanisation en 2007 est estimé à 46\%, ce qui entraîne une forte croissance démographique et une augmentation des besoins alimentaires dans les villes du Bénin (Assogba, 2008). Dans ce contexte la pression parasitaire est une contrainte importante pour les maraîchers pendant la saison des pluies où les ravageurs prédominent. Pour la lutte contre ces ravageurs, les pesticides chimiques spécifiques au maraîchage devraient être utilisés mais compte tenu de leur cherté et rareté, les maraîchers préfèrent utiliser des pesticides chimiques prohibés afin de produire des légumes apparemment sains pour satisfaire la forte demande de la population (Akogbeto et al., 2005; Alhou, 2007). Cette pratique comporte des risques pour la santé et l'environnement tels que la contamination des produits maraîchers, des sols et des eaux par les métaux lourds et autres substances toxiques (Assogba, 2008). L'objectif du présent travail est d'étudier le niveau de contamination des produits maraîchers par les substances toxiques sur le site de Houéyiho en 2014, afin de proposer des mesures pour le minimiser et de mieux appréhender les risques pour la santé et l'environnement, que pourraient engendrer les pratiques maraîchères en vigueur sur le site de Houéyiho au Bénin.

\section{MATERIEL ET METHODES}

Cette étude s'est déroulée sur le site de maraîchage de Houéyiho de Cotonou (Bénin). Il s'agit d'une étude transversale descriptive et analytique menée chez les maraîchers du site de Houéyiho au Bénin en 2014. La méthodologie utilisée est l'approche écosystémique basée sur la transdisciplinarité, la participation, le genre et l'éthique.

La méthode probabiliste a été utilisée pour les cibles (cibles primaires et secondaires) de l'étude. Les cibles primaires ont été les légumes les plus consommés et à maturité prêts pour la consommation; les maraîchers dont les espaces ont été tirés et qui étaient encore présents pendant la période de l'étude constituent les cibles secondaires.

La technique d'échantillonnage à trois degrés a été utilisée pour les cibles primaires :

- Le premier degré consistait à diviser le site de Houéyiho en deux zones (zones Nord et Sud). Chaque zone a été ensuite scindée en deux en fonction du type d'eaux d'arrosage (eau de puits et de marécage). Nous avons procédé au tirage aléatoire, d'un espace dans chaque subdivision des zones. Pour faire le tirage aléatoire nous avons dressé la liste numérotée de tous les espaces (portions de terres) de chaque zone du site de Houéyiho en fonction des eaux d'arrosage, puis tiré au sort un espace (portion de terre) dans chaque subdivision. Nous avons décidé de prendre deux espaces par zone pour des raisons de ressources (temps, finance).

-Le deuxième degré a concerné le choix des planches. Il s'agissait de choisir les planches au niveau des espaces tirés au sort. Pour ce tirage, nous avons dénombré et numéroté les planches par type de légume. Ensuite, nous avons choisi par tirage aléatoire simple pour chaque type de légume une planche pour y prélever les plants à analyser.

-Le troisième degré correspond au tirage de l'unité statistique. Sur chaque planche choisie, nous avons fait trois prélèvements de plants. Un prélèvement a été fait à chaque extrémité de la planche et un autre au milieu de la planche. Ces trois prélèvements mis ensemble représentaient notre unité statistique. Pour les cibles secondaires, la technique aléatoire simple a été utilisée pour le choix des maraîchers à partir de la liste établie.

Nous avons fait un prélèvement par espace et par type de légume soit trois prélèvements au total par espace. Le site ayant été subdivisé en quatre, cela nous donne au total 12 prélèvements. Deux prélèvements d'eau (puits et forage) et deux prélèvements du sol ont été faits dans le cadre de la présente étude. 
Les échantillons d'eau les plus utilisés (puits et forage) sont prélevés à l'intérieur des planches à raison d'un puits ou forage sur trois choisis au hasard entre les zones Nord et Sud. Les échantillons de sol sont prélevés dans les horizons de $0-20 \mathrm{~cm}$ dans la zone racinaire des cultures, et dans les horizons de 20-30 cm au niveau des planches arrosées avec l'eau de puits et l'eau de forage dans les zones Nord et Sud.

Dix pour cent $(10 \%)$ de l'effectif des maraîchers (337) ont été enquêtés, soit 34 maraîchers enquêtés.

Les données recueillies portaient sur deux variables indépendantes relatives aux facteurs environnementaux et aux facteurs liés aux intrants agricoles. La contamination des produits maraîchers a été définie en tenant compte des normes de CODEX STAN 1931995 révisé en 2007 pour les aliments de consommation (Codex Stan, 2007).

Une autorisation a été obtenue auprès du président des maraîchers du site de Houéyiho de Cotonou pour réaliser l'enquête. Après une séance d'explications sur les procédures, le consentement des maraîchers a été obtenu pour la collecte de nos données.

\section{Analyses statistiques}

Le logiciel stata 11 a été utilisé pour l'analyse statistique des données. L'analyse descriptive des variables indépendantes a été faite selon leurs fréquences. Le test de Student a été utilisé pour faire la comparaison des taux moyens de contaminants retrouvés dans les produits maraîchers. Le seuil de signification a été fixé à 0,05 .

\section{RESULTATS}

Au total, 12 prélèvements de légumes, deux prélèvements d'eau, deux prélèvements du sol ont été faits sur le site au cours de l'enquête et 34 maraîchers ont été inclus dans l'étude.

\section{Caractéristiques du site de Houéyiho}

Le site de maraîchage de Houéyiho étudié présente des traits caractéristiques tels que : la présence des marécages dont les eaux sont utilisées pour arroser les produits maraîchers, le plan incliné de la zone Nord par rapport à la zone Sud, et les pratiques culturales inadéquates qui sont autant de facteurs qui influencent les concentrations en métaux lourds. De plus, les ordures ménagères mal triées et contenant des piles, des sachets et autres substances toxiques sont à la base de la présence des métaux comme le fer, le plomb, le cadmium, le cuivre, l'arscenic, le zinc et le mercure, identifiés dans les produits maraîchers.

$\checkmark \quad$ Le Tableau 1 montre que :

-22 maraîchers sur 34 soit 64,7\% utilisent Uré + NPK comme fertilisants agricoles ;

- 28 maraîchers sur 34 soit 82, 4 \% utilisent Lambda comme pesticides

79, 4\% des maraîchers expliquent que l'accélération de la croissance des plants est liée à l'utilisation des engrais chimique sur le site Houéyiho (Tableau 2).

$\checkmark \quad 67,6 \%$ des maraîchers reconnaissent avoir utilisé les eaux de forage et marécage pour l'arrosage de leurs plants (Tableau 3).

$\checkmark \quad$ Le Tableau 4 montre que dans la zone Nord:

- les concentrations en fer, en zinc, en plomb et en mercure sont supérieures dans les laitues arrosées par les eaux de marécage que celles des forages par rapport aux normes ;

- les concentrations en zinc sont supérieures dans les vernonia arrosés par les eaux de marécages que celles des forages par rapport aux normes ;

- les carottes arrosées par les eaux de marécage ont des concentrations supérieures en fer, en zinc et en cuivre par rapport à celles des forages par rapport aux normes.

$\checkmark \quad$ Le Tableau 5 montre une variation de concentration des substances toxiques dosées dans les différents plants quel que soit le type d'arrosage.

$\checkmark$ Il ressort du Tableau 6 une concentration supérieure en fer dans les deux types d'eau utilisée (Eau forage, eau marécage) par rapport aux normes exigées

$\checkmark \quad$ Il ressort du Tableau 7 des concentrations plus élevées en fer, zinc, cuivre 
et plomb dans le sol de la zone Nord par rapport au sol de la zone Sud.

Quelle que soit la zone, les concentrations du sol pour les métaux lourds explorés dépassent largement les valeurs normales.

$\checkmark \quad$ Le Tableau 8 montre dans notre étude :

- dans la zone Sud des concentrations supérieures en fer des plants de vernonia amygdalina à ceux de la zone Nord avec une différence statistiquement significative $(\mathrm{p}<$ $0,03)$;

- dans la zone Nord des concentrations supérieures en zinc des plants de vernonia amygdalina à ceux de la zone du Sud avec une différence statistiquement significative $(\mathrm{p}<$ $0,02)$.

$\checkmark \quad$ Les Tableaux 9 et 10 montrent des concentrations élevées en nitrates dans les zones Sud et Nord selon le type d'eau d'arrosage.

Tableau 1: Répartition des maraîchers selon les types d'engrais et pesticides utilisés.

\begin{tabular}{lcc}
\hline Types d'intrants utilisés & Fréquence & Pourcentage(\%) \\
\hline Uré+NPK & 22 & $\mathbf{6 4 , 7}$ \\
Uré+NPK+Fiente & 3 & 8,8 \\
Uré+NPK+Compost & 9 & 26,5 \\
Total & $\mathbf{3 4}$ & $\mathbf{1 0 0 , 0}$ \\
\hline Lambda & 28 & $\mathbf{8 2 , 4}$ \\
Lambda cale & 1 & 2,9 \\
Huile de nyme & 4 & 11,8 \\
Aucun & 1 & 2,9 \\
Total & $\mathbf{3 4}$ & $\mathbf{1 0 0 , 0}$ \\
\hline
\end{tabular}

Tableau 2 : Répartition des maraîchers selon les raisons qui motivent le choix des engrais utilisés.

\begin{tabular}{lcc}
\hline Choix de l'utilisation des engrais par des maraîchers & Fréquence & Pourcentage (\%) \\
\hline Insuffisance des produits bio & 3 & 8,8 \\
Cherté des produits bio & 4 & 11,8 \\
Accélération de la croissance des plants & 27 & $\mathbf{7 9 , 4}$ \\
Total & $\mathbf{3 4}$ & $\mathbf{1 0 0 , 0}$ \\
\hline
\end{tabular}

Tableau 3 : Répartition des maraîchers en fonction des types d'eau d'arrosage.

\begin{tabular}{lcc}
\hline Source d'eaux utilisées par les maraîchers & Fréquence & Pourcentage(\%) \\
\hline Forage & 3 & 8,8 \\
Marécage & 8 & 23,6 \\
Forage + Marécage & 23 & $\mathbf{6 7 , 6}$ \\
Total & $\mathbf{3 4}$ & $\mathbf{1 0 0 , 0 0}$ \\
\hline
\end{tabular}


V. AGUEH et al. / Int. J. Biol. Chem. Sci. 9(1): 542-551, 2015

Tableau 4 : Concentration des substances toxiques dans les produits maraîchers au niveau de la zone Nord selon le type d'arrosage.

\begin{tabular}{lccccccc}
\hline Produits maraîchers & $\mathbf{F e}(\mathbf{m g} / \mathbf{k g})$ & $\mathbf{Z n ~} \mathbf{( m g} / \mathbf{k g})$ & $\mathbf{C u}(\mathbf{m g} / \mathbf{k g})$ & $\mathbf{A s ~} \mathbf{m g} / \mathbf{k g})$ & $\mathbf{P b}(\mathbf{m g} / \mathbf{k g})$ & $\mathbf{C d}(\mathbf{m g} / \mathbf{k g})$ & $\mathbf{H g}(\mathbf{m g} / \mathbf{k g})$ \\
\hline Laitue forage & 587,713 & 200,000 & 30,671 & $<0,2$ & 3,266 & 0,029 & 0,4000 \\
Laitue marécage & 989,845 & 226,000 & 0,234 & $<0,2$ & 21,648 & 0,197 & 0,5000 \\
Vernonia forage & 384,812 & 204,000 & 14,975 & $<0,2$ & 0,458 & 0,028 \\
Vernonia marécage & 297,600 & 230,000 & 8,519 & $<0,2$ & 4,515 & 0,021 & 0,1990 \\
carotte forage & 1355,033 & 110,000 & 0,089 & 1,200 & 9,032 & 0,075 & 0,2857 \\
Carotte marécage & 892,559 & 131,474 & 1,181 & 0,797 & 8,049 & 0,075 & 0,1471 \\
Valeurs normales & $\mathbf{2}$ & $\mathbf{5}$ & $\mathbf{3}$ & $\mathbf{0 , 1}$ & $\mathbf{0 , 3}$ & $\mathbf{0 , 2}$ & $\mathbf{0 , 4}$ \\
\hline
\end{tabular}

Tableau 5 : Concentration des substances toxiques dans les produits maraîchers au niveau de la zone Sud selon le type d'arrosage.

\begin{tabular}{|c|c|c|c|c|c|c|c|}
\hline Nature de l'échantillon & $\mathrm{Fe}(\mathrm{mg} / \mathrm{kg})$ & $\mathrm{Zn}$ (mg/kg) & $\mathrm{Cu}(\mathrm{mg} / \mathrm{kg})$ & As (mg/kg) & $\mathrm{Pb}(\mathrm{mg} / \mathrm{kg})$ & $\mathrm{Cd}(\mathrm{mg} / \mathrm{kg})$ & Hg (mg/kg) \\
\hline Laitue forage & 1912,424 & 133,733 & 0,173 & $<0,2$ & 25,414 & 0,146 & 0,3546 \\
\hline Laitue marécage & 679,355 & 235,644 & 12,982 & $<0,2$ & 21,649 & 0,051 & 0,1471 \\
\hline Vernonia forage & 1199,169 & 130,682 & 14,858 & $<0,2$ & 7,305 & 0,059 & 0,1579 \\
\hline Daucus carota forage & 428,886 & 96,899 & 0,785 & $<0,2$ & 3,435 & 0,891 & 0,1970 \\
\hline Daucus carota marécage & 539,663 & 98,000 & 1,048 & $<0,2$ & 6,317 & 0,708 & 0,1395 \\
\hline
\end{tabular}


Tableau 6 : Concentration de substances toxiques selon le type d'eau utilisée.

\begin{tabular}{lccccccc}
\hline Nature des échantillons & $\begin{array}{c}\mathbf{F e} \\
(\mathbf{m g} / \mathbf{k g})\end{array}$ & $\begin{array}{c}\mathbf{Z n} \\
(\mathbf{m g} / \mathbf{k g})\end{array}$ & $\begin{array}{c}\mathbf{C u} \\
(\mathbf{m g} / \mathbf{k g})\end{array}$ & $\begin{array}{c}\mathbf{A s} \\
(\mathbf{m g} / \mathbf{k g})\end{array}$ & $\begin{array}{c}\mathbf{P b} \\
(\mathbf{m g} / \mathbf{k g})\end{array}$ & $\begin{array}{c}\mathbf{C d} \\
(\mathbf{m g} / \mathbf{k g})\end{array}$ & $\begin{array}{c}\mathbf{H g} \\
(\mathbf{m g} / \mathbf{k g})\end{array}$ \\
\hline Eau forage & 4,410 & 1,050 & 0,002 & $<0,001$ & 0,018 & 0,001 & 0,0045 \\
Eau marécage & 4,160 & 0,950 & 0,002 & $<0,001$ & 0,107 & 0,013 & 0,0003 \\
Valeurs normales & $\mathbf{2}$ & $\mathbf{5}$ & $\mathbf{3}$ & $\mathbf{0 , 1}$ & $\mathbf{0 , 3}$ & $\mathbf{0 , 2}$ & $\mathbf{0 , 4}$ \\
\hline
\end{tabular}

Tableau 7: Concentration de substances toxiques du sol au niveau des zones Nord et Sud.

\begin{tabular}{lccccccc}
\hline Nature des échantillons & $\begin{array}{c}\mathbf{F e} \\
(\mathbf{m g} / \mathbf{k g})\end{array}$ & $\begin{array}{c}\mathbf{Z n} \\
(\mathbf{m g} / \mathbf{k g})\end{array}$ & $\begin{array}{c}\mathbf{C u} \\
(\mathbf{m g} / \mathbf{k g})\end{array}$ & $\begin{array}{c}\mathbf{A s} \\
(\mathbf{m g} / \mathbf{k g})\end{array}$ & $\begin{array}{c}\mathbf{P b} \\
(\mathbf{m g} / \mathbf{k g})\end{array}$ & $\begin{array}{c}\mathbf{C d} \\
(\mathbf{m g} / \mathbf{k g})\end{array}$ & $\begin{array}{c}\mathbf{H g} \\
(\mathbf{m g} / \mathbf{k g})\end{array}$ \\
\hline Zone Sud & 5750,090 & 294,000 & 7,939 & 2,347 & 20,732 & 1,861 & 0,4798 \\
Zone Nord & 11302,990 & 3944,773 & 43,291 & 4,438 & 91,404 & 1,802 & 0,5480 \\
Valeurs normales & $\mathbf{2}$ & $\mathbf{5}$ & $\mathbf{3}$ & $\mathbf{0 , 1}$ & $\mathbf{0 , 3}$ & $\mathbf{0 , 2}$ & $\mathbf{0 , 4}$ \\
\hline
\end{tabular}


V. AGUEH et al. / Int. J. Biol. Chem. Sci. 9(1): 542-551, 2015

Tableau 8: Comparaison des concentrations des substances toxiques des produits maraîchers en fonction des zones Nord et Sud.

\begin{tabular}{lcccccc}
\hline Produits maraîchers & Fe $(\mathbf{m g} / \mathbf{k g})$ & $\mathbf{Z n}(\mathbf{m g} / \mathbf{k g})$ & $\mathbf{C u}(\mathbf{m g} / \mathbf{k g})$ & $\mathbf{P b}(\mathbf{m g} / \mathbf{k g})$ & $\mathbf{C d}(\mathbf{m g} / \mathbf{k g})$ & $\mathbf{H g}(\mathbf{m g} / \mathbf{k g})$ \\
\hline $\begin{array}{l}\text { Vernonia amygdalina } \\
\text { Zone Nord }\end{array}$ & 341,2060 & 217,000 & 11,7470 & 2,4865 & 0,0245 & 0,2424 \\
\hline $\begin{array}{l}\text { Vernonia amygdalina } \\
\text { Zone Sud }\end{array}$ & 1074,0215 & 122,9950 & 12,7525 & 8,2795 & 0,0465 & 0,1415 \\
\hline $\boldsymbol{P}$ value & $\mathbf{0 , 0 3}$ & $\mathbf{0 , 0 2}$ & 0,81 & 0,12 & 0,23 & 0,16 \\
\hline $\begin{array}{l}\text { Lactuca sativa } \\
\text { Zone Nord }\end{array}$ & $\mathbf{7 8 8 , 7 7 9 0}$ & 213,000 & 15,4525 & 12,4570 & 0,1130 & 0,4500 \\
\hline $\begin{array}{l}\text { Lactuca sativa } \\
\text { Zone Sud }\end{array}$ & 1295,8895 & 184,6885 & 6,5775 & 23,5300 & 0,0985 & 0,2509 \\
\hline $\boldsymbol{P}$ value & 0,51 & 0,64 & 0,64 & 0,34 & 0,89 & 0,23 \\
\hline $\begin{array}{l}\text { Daucus carota } \\
\text { Zone Nord }\end{array}$ & 1123,7960 & 120,7370 & 0,6350 & 8,5405 & 0,0750 & 0,1421 \\
\hline $\begin{array}{l}\text { Daucus carota } \\
\text { Zone Sud }\end{array}$ & 484,2745 & 97,4495 & 0,9165 & 3,1675 & 0,3545 & 0,1683 \\
\hline $\boldsymbol{P}$ value & 0,11 & 0,16 & 0,66 & 0,07 & 0,09 & 0,46 \\
\hline
\end{tabular}

Tableau 9 : Concentration des produits maraîchers en nitrates au niveau de la zone Sud selon le type d'arrosage.

\begin{tabular}{lllll}
\hline Produits maraichers & $\mathbf{\%} \mathbf{M S}$ & $\mathbf{\%} \mathbf{~ H}$ & $\mathbf{N O}_{\mathbf{3}}{ }^{-} \mathrm{mg} / \mathrm{kg}$ & $\mathbf{N}^{-N_{\mathbf{3}}{ }^{-} \mathrm{mg} / \mathrm{kg}}$ \\
\hline Laitue forage & 9,66 & 90,34 & 6038,65 & 1380,26 \\
Laitue marécage & 12,16 & 87,84 & 5208,33 & 1233,55 \\
Vernonia forage & 14,12 & 85,88 & 3895,18 & 826,25 \\
Vernonia marécage & 13,4 & 86,6 & 7711,44 & 1741,29 \\
Carotte forage & 10,46 & 89,54 & 5895,47 & 1274,70 \\
Carotte marécage & 11,27 & 88,73 & 6654,84 & 1478,85 \\
\hline
\end{tabular}

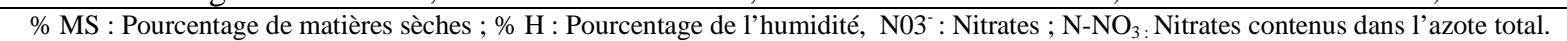


Tableau 10 : Concentration des produits maraîchers en nitrates au niveau de la zone Nord selon le type d'arrosage.

\begin{tabular}{lllll}
\hline $\begin{array}{l}\text { Produits } \\
\text { maraîchers }\end{array}$ & \% $\mathbf{M S}$ & $\mathbf{\%} \mathbf{H}$ & $\begin{array}{l}\mathbf{N O}_{3}^{-} \\
\mathrm{mg} / \mathrm{kg}\end{array}$ & $\mathbf{N}-\mathbf{N O}_{\mathbf{3}}{ }^{-} \mathrm{mg} / \mathrm{kg}$ \\
\hline Laitue forage & 8,76 & 91,24 & 9132,42 & 2092,85 \\
Laitue marécage & 8,18 & 91,82 & 10594,95 & 2444,99 \\
Vernonia forage & 13,16 & 86,84 & 6585,61 & 1519,76 \\
Vernonia marécage & 29,1 & 70,9 & 4639,18 & 1030,93 \\
Carotte forage & 10,27 & 89,73 & 6653,68 & 1460,56 \\
Carotte marécage & 9,97 & 90,03 & 7856,90 & 1838,85 \\
\hline
\end{tabular}

$\%$ MS : Pourcentage de matières sèches ; \% H : Pourcentage de l'humidité, $\mathrm{N} 03^{-}$: Nitrates

$\mathrm{N}-\mathrm{NO}_{3}$ : Nitrates contenus dans l'azote total.

\section{DISCUSSION}

L'analyse minérale des sept espèces végétales montre de fortes teneurs pour les éléments suivants : Cuivre $(\mathrm{Cu}), \mathrm{Fer}(\mathrm{Fe})$, mercure $(\mathrm{Hg})$, Cadmium $(\mathrm{Cd})$, Plomb $(\mathrm{Pb})$, Zinc (Zn), et Arsenic (As). Comparativement aux normes de concentrations préconisées par l'OMS pour les produits de consommation, les excès de concentration sont supérieurs à 1000 $\mathrm{mg} / \mathrm{kg}$ pour le $\mathrm{Fe}$, et sont compris entre 10 et $100 \mathrm{mg} / \mathrm{kg}$ pour le $\mathrm{Pb}, \mathrm{Cu}, \mathrm{As}, \mathrm{Hg}, \mathrm{Cd}, \mathrm{Zn}$. La classification des teneurs en éléments métalliques par ordre d'importance varie avec les différentes espèces végétales. Les végétaux à croissance rapide comme les légumes sont connus pour leur aptitude à l'accumulation des métaux lourds (Harmanescu et al., 2011). Cependant, lorsque les taux d'accumulation deviennent élevés, ces éléments deviennent polluants. La présente étude montre que les produits maraîchers cultivés sur le site de maraîchage de Houéyiho sont fortement pollués par des métaux lourds. Les ordures ménagères mal triées et contenant des piles, des sachets et autres substances toxiques sont à la base de la présence des métaux comme le fer, le plomb, le cadmium, le cuivre, l'arsenic, le zinc et le mercure, identifiés dans les produits maraîchers. Les études réalisées par Pazou Yehouenou et al. (2010) au Bénin ont montré des concentrations en fer, zinc, plomb, et cuivre largement en dessous de celles que nous avons réalisées en 2014 ; cela s'explique par le fait que le niveau de contamination depuis 2010 à ce jour a fortement augmenté sur le site de Houéyiho. Le taux élevé de ces métaux serait également dû à l'utilisation de façon abusive des produits phytosanitaires et aussi à la mauvaise qualité des eaux d'arrosage.

Les substances toxiques sont davantage accumulées dans les légumes feuilles que dans les légumes à tubercules. Il est démontré que chez les végétaux, certains éléments $(\mathrm{Fe}, \mathrm{Cd})$ absorbés à partir du sol s'accumulent de préférence dans les feuilles (Harmanescu et al., 2011; Temple et Moustier, 2004). Cependant, tous les éléments accumulés dans les feuilles ne proviennent pas nécessairement du sol. Ils peuvent pénétrer directement dans les feuilles à partir de l'atmosphère (Caille, 2002; Bonnard, 2005). Dans cette étude, le fait que la majorité des substances toxiques analysées se retrouvent plus concentrées dans les feuilles que dans les tubercules pourrait indiquer une absorption des éléments essentiellement par voie foliaire à partir de l'atmosphère surtout quand on sait que le site de Houéyiho se situe sur la trajectoire des avions en voie d'atterrissage. En outre, les feuilles pourraient aussi accumuler directement certains métaux lourds associés aux pesticides pulvérisés sur les plantes (Moulton et al., 2000).

Le taux élevé du plomb et du cadmium dans le sol s'explique par la présence des piles et batteries usagées, retrouvées dans le 
compost suite à un mauvais tri à la base avant l'enfouissement. Nous avons aussi noté des teneurs élevées en nitrates et ammonium dont les valeurs sont supérieures aux normes exigées par la norme CODEX STAN 1931995 révisé en 2007 (Codex Stan, 2007). Ce qui expliquerait leur accumulation dans les déchets. La teneur très élevée de fer dans les produits maraîchers (vernonia: 707,61 $\mathrm{mg} / \mathrm{kg}$; laitue $1042,33 \mathrm{mg} / \mathrm{kg}$; daucus carota $804,03 \mathrm{mg} / \mathrm{kg}$ ) se justifie par la forte proportion de matières ferreuses présentes dans les ordures ménagères utilisées comme compost (aiguilles, boîtes de conserve, etc.). Ces résultats confirment celui de Sanny (2002) sur les bio-contaminants et migration des agents toxiques dans les cultures maraîchères au Bénin. Les concentrations de zinc $(42 \mathrm{mg} / \mathrm{kg}$ à $185 \mathrm{mg} / \mathrm{kg})$ et de plomb $(1,65$ $\mathrm{mg} / \mathrm{kg}$ à $4,85 \mathrm{mg} / \mathrm{kg}$ ) obtenues dans le sol du site maraîcher de Houéyiho en 2010 par Pazou Yehouenou et al. (2010) sont inférieures à celles obtenues dans notre étude en 2014 et dans l'étude de Gnandli et al. (2006) dans les sols des sites maraîchers prélevés le long de l'autoroute Lomé-Aného au sud du Togo (Gnandli et al., 2006).

Les concentrations en fer $(1042,33$ $\mathrm{mg} / \mathrm{kg})$, zinc $(198,844 \mathrm{mg} / \mathrm{kg})$, cuivre $(11,02$ $\mathrm{mg} / \mathrm{kg})$, et plomb $(17,99 \mathrm{mg} / \mathrm{kg})$ obtenues dans les laitues cultivées sur le site maraîcher de Houéyiho au Bénin en 2014 sont supérieures à celles obtenues dans les laitues cultivées le long de l'autoroute Lomé-Aného au sud du Togo (Gnandli et al., 2013) . Ces différentes concentrations observées dans les différentes études réalisées expliquent une dégradation des pratiques culturales de Houéyiho par rapport à l'année 2010 et celles des autres sites de maraîchage.

\section{Conclusion}

Cette étude a montré que le niveau de contamination des produits marâichers par les métaux lourds a augmenté avec des concentrations variées par rapport à 2010 . Néanmoins, ces diverses concentrations sont en général meilleures pour certains métaux lourds (Cadmium, Mercure, Arsenic) à
Houéyiho qu'au niveau d'autres sites maraîchers. Des efforts restent à fournir pour réduire davantage ces concentrations à travers des sensibilisations pour l'utilisation effective, la disponibilité et l'accessibilité des intrants biologiques.

L'amélioration des résultats obtenus passe par l'élaboration d'un plan tactique dont la mise en œuvre conséquente permettra le renforcement des acquis qui serviront de gage pour le passage à l'échelle nationale.

\section{REFERENCES}

Akogbeto M, Djouaka R, Noukpo H.2005. Utilisation des insecticides agricoles au Bénin. Bulletin de la Société de Pathologie Exotique, 12(98) :400-405.

Alhou B. 2007. Impact des rejets de la ville de Niamey (Niger) sur la qualité des eaux du fleuve Niger. Thèse de Doctorat en Science, Université Libre de Bruxelles en Belgique, 230p.

Assogba B. 2008. Typologie des exploitations maraîchères au Sud-Benin. Mémoire D.I.T, Université d'Abomey-Calavi, Bénin, 102 p.

Bonnard R. 2005. Impact des incertitudes liées aux coefficients de transfert dans les évaluations de risque sanitaire. Rapport d'étude INERIS (ministère français de l'Environnement), $26 \mathrm{p}$.

Caille N. 2002. Mobilité et phytodisponibilité du mercure dans les sédiments de curage. Thèse de Doctorat en Science, ENSAIA, Vandœuvre-lès-Nancy, 167p.

Codex Stan. 2007. Norme générale pour les contaminants et les toxines des aliments. Codex Stan 193-1995 Révisé.

Gnandli K, Koffi T, Aléodjrodo E, Hazou A, Koffi A, Komi A et al. 2013. Bioaccumulation de certains éléments métalliques dans les produits maraîchers cultivés sur les sols urbains le long de l'autoroute Lomé-Aného, Sud Togo Laboratoire Gestion et traitement des déchets (GTVD), Faculté des sciences , Université de Lomé http://www. tandfonline.com/loi/tabg20. 
Gnandli K, Tozo K, 2Edorh AP, Abi, Agbeko $\mathrm{K}$, Amouzouvi K, Baba G, Tchangbedji G, Killi K. 2006. La Bioaccumulation du plomb et autres métaux lourds dans les produits maraîchers cultivés sur les sols urbains le long de l'autoroute LoméAného, Sud Togo. Journal of African Earth Sciences, 37: 1-2.

Harmanescu M, Alda LM, Bordean DM, Ioan Gogoasa L and Gergen L. 2011.Heavy metals health risk assessment for population via consumption of vegetables grown in old mining area; a case study: Banat County,Romania. Chemistry Central Journal, 5(64): 2-10.

Madjouma K, Kpérkouma W, Komlan B, Gbandi DB, Adam A, Koffi A. 2009. Le maraîchage périurbain à Lomé pratiques culturales, risques sanitaires et dynamiques spatiales. Cah. Agric., 18(4): 356-363.

Moulton L, West J, Berner A. 2000. Solute flux and mineral mass alance approaches to the quantification of plant effect on silicate weathering. Amer. J. Sci., 300: 539-570.

Pazou Yehouenou EA, Soton A, Azocli A, Acakpo H, BoKo M, Fourn L, Houinsa D, Keke J-C, Fayomi B. 2010. Contamination du sol, de l'eau et des produits maraîchers par des substances toxiques et des métaux lourds sur le site de Houéyiho (Cotonou) en République du Bénin. Int. J. Biol. Chem. Sci., 4(6): 2160-2168.

Sanny MS. 2002. Etude des bio-contaminants et migration des agents toxiques dans les cultures maraîchères : cas du périmètre maraîcher de Houéyiho à Cotonou. Mémoire D.I.T EPAC, Université d'Abomey Calavi, Bénin, 102 p.

Temple L, Moustier P. 2004. Les fonctions et contraintes de l'agriculture périurbaine de quelques villes africaines (Yaounde', Cotonou, Dakar). Cah. Agric., 13:15-22. 